ERRATUM

\title{
Space and time in visual context
}

Odelia Schwartz, Anne Hsu and Peter Dayan

Nature Reviews Neuroscience 8, 522-535 (2007)

On page 532 and 533 of the above article, the author of references 17 and 70 was given incorrectly. These references should have read:

17. Zhaoping, L. Border ownership from intracortical interactions in visual area V2. Neuron 47, 143-153 (2005).

70. Zhaoping, L. Theoretical understanding of the early visual processes by data compression and data selection. Network 17, 301-334 (2006).

Also, on page 535, in the Further information box, the authors' names were misspelled. The box should have read:

Odelia Schwartz's homepage: http://neuroscience.aecom.yu.edu/faculty/primary faculty pages/schwartz.html Peter Dayan's homepage: http://www.gatsby.ucl.ac.uk/ dayan/ 